\title{
Assessment of constant-potential implicit solvation calculations of electrochemical energy barriers
}

\author{
Maxime Van den Bossche, ${ }^{\dagger}+$ Egill Skúlason, ${ }^{\ddagger}$ Christoph Rose-Petruck, ${ }^{\dagger}$ and \\ Hannes Jónsson*,‡ \\ $\dagger$ Department of Chemistry, Brown University, Providence, RI, United States \\ $\ddagger$ Science Institute and Faculty of Physical Sciences, University of Iceland, 107 Reykjavik, \\ Iceland \\ E-mail: hj@hi.is
}

\section{Abstract}

Theoretical estimation of the activation energy of electrochemical reactions is of critical importance but remains challenging. In this work, we address the usage of an implicit solvation model for describing hydrogen evolution reaction steps on $\operatorname{Pt}(111)$ and $\operatorname{Pt}(110)$, and compare with the 'extrapolation' approach and coworkers as well as single-crystal measurements. We find that both methods yield qualitatively similar results, which are in fair agreement with the experimental data. Care should be taken, however, in addressing spurious electrostatic interactions between periodically repeated slabs in the VASPsol implementation. Considering the lower computational cost and higher flexibility of the implicit solvation approach, we expect this method to become a valuable tool in electrocatalysis.

\section{Introduction}

Electrocatalytic reactions involve electron transfer to or from an electrode surface and are evidently crucial for all electrochemical production processes, as well as for corrosion chemistry and the electronics industry. Detailed insights into the reaction mechanism and kinetics, however, are difficult to obtain and generally require a combination of experimen- 28 tal and computational work.

While in classical, "gas phase" heterogeneous 30 catalysis the field of theoretical modeling is 31 rather well established, further methodologi- 32 cal development is still required when it comes 33 to electrocatalysis. Two major challenges in 34 this field are given by (i) the description of 35 metal/electrolyte interface, and (ii) the require- $\quad 36$ ment of constant electrode potential over the 37 course of an electron transfer reaction. These 38 aspects complicate, in particular, the evalua- 39 tion of activation energies of electron trans- 40 fer reactions. As a result, the vast majority ${ }_{41}$ of computational electrocatalysis studies have 42 been limited to thermodynamic properties only, $\quad 43$ such as reaction energies. ${ }^{1,2}$ It is, however, well 44 recognized that the proper study of reaction 45 kinetics (in electrocatalysis as in conventional 46 catalysis) requires knowledge of transition state $\quad 47$ energies. $^{3,4}$

Several approaches have so far been proposed to address these challenges, which differ especially in the manner the constant-potential requirement is handled. This requirement stipulates that the electrode potential w.r.t. the reference electrode remains constant over the course of an elementary reaction, also when it is accompanied by electron transfer. As only a ${ }_{56}$ finite number of electrons $N_{\mathrm{e}}$ can be included in $\quad{ }_{57}$ 
the electronic structure calculations, however, the potential will change significantly over the course of an electron transfer reaction, if $N_{\mathrm{e}}$ is held constant. ${ }^{5-7}$ Extended electrode surfaces are furthermore most conveniently modeled using periodically repeated supercells, which must satisfy overall charge neutrality.

In this context, the arguably most rigorous method is the so-called 'extrapolation' approach, ${ }^{5,6}$ where energy barriers are evaluated at successively larger lateral cell dimensions and extrapolated to the infinite cell limit where the potential drop is zero. The number of electrons $N_{\mathrm{e}}$ is determined by the charge neutrality condition, which entails that e.g. hydrogen atoms must be added to or withdrawn from the electrolyte to vary the electrode potential. This method has so far exclusively been applied to FCC(111) surfaces in conjunction with ice-like hexagonal water structures. ${ }^{5,6,8,9}$

In a second, related method, ${ }^{10,11}$ the computationally costly extrapolation is avoided by assuming that the required correction to the transition state energy has a purely capacitive character. This correction is based on changes in the charges of the metal atoms at the electrode surface, and hence an additional ambiguity lies in the choice of the charge density partitioning scheme.

Thirdly, in the 'double reference' approach $^{12,13}$ the electrode potential is controlled by varying $N_{\mathrm{e}}$, with introduction of a homogeneous compensating background to maintain overall charge neutrality. It is not quite clear, at this point, if this method applies sound corrections to the obtained total energies, which is known to be a non-trivial problem in the case of charged slabs in homogeneous compensating backgrounds. ${ }^{14,15}$

Methods have also been proposed where charge neutrality is maintained by localizing the compensating charge in a 'counterelectrode' at some distance away from the electrode/electrolyte interface. ${ }^{16,17}$ In reality, however, charge compensation is mediated by changes in the concentration profiles of the electrolyte's ions near the electrode surface.

In yet another approach ${ }^{18,19}$ electrochemical barriers are estimated in a two-step pro- cedure inspired by Markus theory. First, the ${ }_{107}$ activation energy of the corresponding non- 108 electrochemical reaction is calculated (e.g. reaction with adsorbed $\mathrm{H}$ in the case of a protoncoupled electron transfer ( $\mathrm{PCET}$ ) reaction) and it is assumed that, at the same potential, the electrochemical process possesses the same activation energy. The potential dependence of the activation energy is then described using e.g. Butler-Volmer theory, requiring further simplifying assumptions regarding the symmetry factor of the reaction.

Finally, a more recently developed approach, which we will be focusing on in this work, is one where (part of) the electrolyte is approximated by a polarizable dielectric continuum (i.e. an implicit solvation model). ${ }^{20-23}$ The constant-potential requirement is here obeyed by varying $N_{\mathrm{e}}$ with concomitant changes in the concentration of counterions in the surrounding electrolytes (frequently described by Poisson-Boltzmann theory). The main assumption here, then, is that the ionic distribution in the electrolyte is equilibrated also at the saddle point, and not only at the reactant and product states.

Although several studies have applied the above constant-potential approach with implicit solvation models (using e.g. the VASPsol, ${ }^{24-27}$ JDFTx, ${ }^{28}$ SIESTA $^{29-33}$ and GPAW codes $^{34}$ ), we are not aware of attempts to compare its results to those of the extrapolation method, or to detailed single-crystal measurements.

The present work offers such a comparison, focusing on the various steps of the hydrogen evolution reaction (HER) on $\mathrm{Pt}(111)$ and $\mathrm{Pt}(110)$. There are three types of reactions relevant to the HER:

$$
\begin{array}{ll}
\text { Volmer: } & \mathrm{H}^{+}+\mathrm{e}^{-}+^{*} \rightarrow \mathrm{H}^{*} \\
\text { Tafel: } & 2 \mathrm{H}^{*} \rightarrow \mathrm{H}_{2}+2^{*} \\
\text { Heyrovský: } & \mathrm{H}^{+}+\mathrm{e}^{-}+\mathrm{H}^{*} \rightarrow \mathrm{H}_{2}+^{*}
\end{array}
$$

Adsorbed $\mathrm{H}$ and vacant adsorption sites are de- ${ }_{141}$ noted $\mathrm{H}^{*}$ and ${ }^{*}$, respectively.

The (111) facet is chosen to compare with the extrapolation approach, which has so far been exclusively applied to FCC(111) surfaces due to 
(a) Protonated ice bilayer

(b) Eigen ion

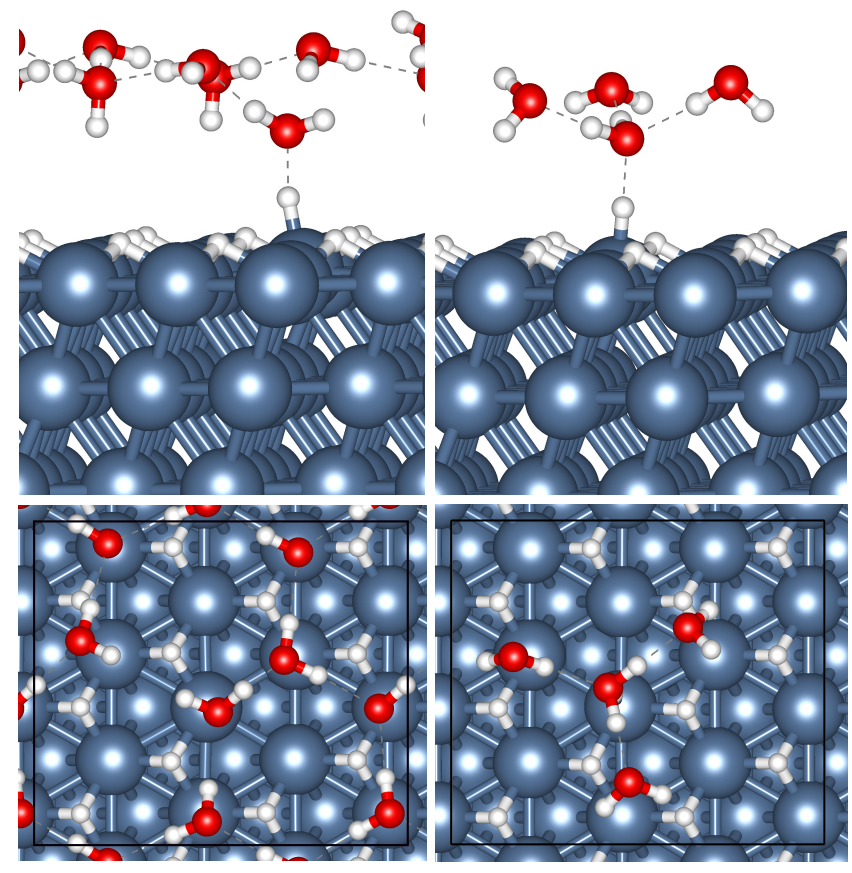

Figure 1: Side and top views of the obtained transition states for the Volmer reaction on $\mathrm{Pt}(111)$ at an initial hydrogen coverage of 0.92 ML. The considered water structure are a protonated hexagonal ice bilayer [Panel (a)] and a $\mathrm{H}_{3} \mathrm{O}^{+}\left(\mathrm{H}_{2} \mathrm{O}\right)_{3}$ ('Eigen') cation [Panel (b)]. the commensurability with the hexagonal ice bilayer structure used to model the metal/water interface. The structure of this adlayer is illustrated in Figure 1, in the transition state for hydrogen deposition via the Volmer reaction. As the implicit solvation approach is less restricted in terms of water structures, we will also compare with results from a water cluster model, corresponding to the 'Eigen' cation in its protonated form $\left(\mathrm{H}_{3} \mathrm{O}^{+}\left(\mathrm{H}_{2} \mathrm{O}\right)_{3}\right)$. Also the HER steps on (110) facets are included, as experimental Tafel slopes are here easier to analyze and indicate a Volmer-Tafel mechanism with the Tafel reaction as the rate-determining step (RDS). ${ }^{35}$

It should be noted that the above considerations apply not only to activation energies but also to reaction energies. The latter have, however, typically been calculated using the computational hydrogen electrode (CHE) formalism, ${ }^{1}$ which makes use of the equilibrium relation of the proton/hydrogen redox-couple of the standard hydrogen electrode (SHE). The focus of this work will hence lie on the estimation of 169 barrier heights.

\section{Computational methods}

To facilitate the comparison to results from 172

previous publications with the extrapolation 173 approach, ${ }^{6}$ the same computational setup is used here. The bulk of the electronic structure calculations are performed with the VASP code ${ }^{36-39}$ using Kohn-Sham density functional theory (DFT). ${ }^{40,41}$ Core electrons are treated via standard projector augmented wave (PAW) setups with the following valences: $\mathrm{H}(1), \mathrm{O}(6)$, $\mathrm{Pt}(10)$. The basis set consists of plane waves with a kinetic energy up to $350 \mathrm{eV}$.

Additional calculations are carried out using the JDFTx code ${ }^{21}$ using GBRV ultrasoft pseudopotentials $^{42}$ with the recommended cutoffs for the wave functions (20 Hartree) and the electron density (100 Hartree). ${ }^{1}$

Electronic exchange and correlation are described using the revised Perdew-BurkeErnzerhof (RPBE) functional ${ }^{43}$ which modifies the exchange enhancement factor of the original PBE expression ${ }^{44}$ to improve the description of adsorption energies on transition metal surfaces. In JDFTx the RPBE functional is implemented through the LibXC library. ${ }^{45}$

Three-layer slabs of $c(3 \times 4)$ geometry are employed for the $\operatorname{Pt}(111)$ surface, with the RPBE crystal lattice constant of $4.025 \AA$. For the $\mathrm{Pt}(110)$ slab, six metal layers are used with a $p(2 \times 4)$ cell size and a $(1 \times 2)$ missing-row reconstruction. In both cases the first Brillouin zone is sampled using $(4 \times 3 \times 1)$ MonkhorstPack grids. ${ }^{46,47}$ Local minimizations and saddle point searches with the dimer method ${ }^{48,49}$ are pursued until the largest force components are less than $0.05 \mathrm{eV} / \AA$ in magnitude. All metal layers except the topmost layer are constrained to their bulk lattice positions. The distances separating periodically repeated slabs will be addressed in the Results section.

Implicit solvation calculations of the aqueous electrolyte are performed using the GLSSA13 174 175 176 177 178 179 180 181

\footnotetext{
${ }^{1}$ In the GBRV pseudopotential set the $5 p^{6}$ semicore states are included in the valence for Pt.
} 
solvent model, ${ }^{50}$ which is available in VASP through the VASPsol extension. ${ }^{22,23}$ Like other models such as SCCS, ${ }^{51}$ the method puts forward a dielectric profile $\epsilon$ as a functional of the Kohn-Sham electron density distribution $\rho$ :

$$
\epsilon[\rho]=1+\left(\epsilon_{\text {bulk }}-1\right) S[\rho],
$$

with the following form for the 'shape function' $S$ :

$$
S[\rho]=\frac{1}{2} \operatorname{erfc}\left(\frac{\log \left(\rho / \rho_{\text {cut }}\right)}{\sigma \sqrt{2}}\right) .
$$

The dielectric function therefore changes gradually from 1 to the bulk dielectric constant $\epsilon_{\text {bulk }}$ (78.4, the experimental value for liquid water at $\left.298 \mathrm{~K}^{52}\right)$. The particular shape of this transition is governed by the $\sigma$ and $\rho_{\text {cut }}$ parameter, for which we have applied the default values (0.6 and $0.0025 \AA^{-3}$, respectively). The free energy required to create a solvent cavity around the solute is calculated as

$$
\mathrm{A}_{\text {cavity }}=\tau \int|\nabla S| d \mathbf{r}
$$

where the $\tau$ parameter $\left(0.525 \mathrm{meV} / \AA^{2}\right)$ has been optimized to reproduce the solvation energies of a series of organic molecules. ${ }^{22}$

In case the electrode potential is different from the potential of zero charge (PZC), a counterion charge distribution is included in the implicit solvent region through the use of the generalized Poisson-Boltzmann equation:

$\nabla \cdot \epsilon(\mathbf{r}) \nabla \phi_{t o t}(\mathbf{r})=\left(\frac{2 c_{0} q^{2} e^{2}}{k_{B} T}\right) \phi_{t o t}(\mathbf{r})-\rho_{\text {solute }}(\mathbf{r})$

$c_{ \pm}(\mathbf{r})=c_{0} \exp \left(\frac{ \pm q e \phi_{t o t}(\mathbf{r})}{k_{B} T}\right) S[\rho(\mathbf{r})]$.

We used $\kappa$ values corresponding to a Debye length of $3 \AA$, corresponding to a $1 \mathrm{M}$ concentration of a 1:1 electrolyte $\left(c_{0}=1 \mathrm{M}\right)$. This mimics the experimental situation where $1 \mathrm{M}$ of a strong acid is used ( $\mathrm{U}_{\mathrm{SHE}}=0$ for HER and $\mathrm{pH}=0)$.

Though grand-canonical SCF algorithms exist $^{21}$ which vary the number of electrons $N_{e}$ so as to match the targeted chemical potential, currently only conventional (canonical) meth- 246 ods are available in VASPsol, making it nec- 247 essary to adjust $N_{e}$ in between successive SCF ${ }_{248}$ loops instead. For local minimization runs, we 249 use a simple iterative approach:

$$
N_{e}(i+1)=N_{e}(i)-a \cdot\left[\mu_{e}(i)-\mu_{e, \text { target }}\right] .
$$

We find an $a$ value of $1.0 \mathrm{~V}^{-1}$ to be appropriate ${ }_{251}$ for the structures considered in this work. In 252 this manner, the electrode potential converges $\quad 253$ to the targeted value as the local optimization 254 proceeds. For saddle point searches, we find it 255 more convenient to adapt $N_{e}$ after a completed $\quad 256$ dimer search, iterating until the target potential ${ }_{257}^{256}$ is matched within $15 \mathrm{mV}$.

The electronic chemical potential $\mu_{e}$ is calculated by comparing the Fermi level with the potential in the bulk electrolyte, which in an implicit solvent equals that of an electron in vacuum: ${ }^{23}$

$$
\mu_{e}=\epsilon_{\mathrm{Fermi}}-V_{\mathrm{bulk}}
$$

If, then, a certain potential $U$ is to be attained 264 w.r.t. the standard hydrogen electrode (SHE), 265 the target chemical potential is given by:

$$
\mu_{e, \text { target }}=-\Phi_{\mathrm{SHE}}-U,
$$

where $\Phi_{\mathrm{SHE}}$ is the work function of the SHE. ${ }_{267}$ We have taken $\Phi_{\text {SHE }}$ equal to $4.43 \mathrm{~V}$, which lies 268 within the experimental value of the SHE work 269 function compared to vacuum which is usually 270 measured to be $4.44(+/-0.02) \mathrm{V} .^{53}{ }^{271}$

To compare energy differences of two struc- ${ }_{272}$ tures at different $N_{e}$ values (but identical elec- 273 trode potential $U$ ), the VASPsol and JDFTx ${ }_{274}$ output energies need to be corrected by the cost 275 (gain) of removing (adding) electrons:

$$
\Omega=E_{\mathrm{DFT}}+N_{\mathrm{e}} \mu_{\mathrm{e}}(U) .
$$

Even though $E_{\mathrm{DFT}}$ here includes entropy terms $\quad 277$ from the solvation model, we will refer to $\Delta \Omega$ as 278 (electronic) grand-canonical energy differences, 279 as no entropic contributions are included from 280 the explicit atom calculations.

The symmetry factor $\beta$ for an elementary re- 282 action at a given $U$ corresponds to the deriva- ${ }_{283}$ 
tive of the activation energy w.r.t. $U$, which we evaluate using a central difference scheme $(\Delta U=0.1 \mathrm{~V})$

$$
\begin{aligned}
\beta(U) & =\frac{\partial \Delta \Omega_{\mathrm{act}}}{e \partial U}, \\
& \simeq \frac{\Delta \Omega_{\mathrm{act}}(U+\Delta U)+\Delta \Omega_{\mathrm{act}}(U-\Delta U)}{2 e \Delta U} .
\end{aligned}
$$

\section{Results and Discussion}

In the following paragraphs we present our results for Volmer, Tafel, and Heyrovský energy barriers on $\operatorname{Pt}(111)$ and $\operatorname{Pt}(110)$ at $U=0 \mathrm{~V}$ on the SHE scale.

\section{$\mathrm{H}_{2}$ evolution on $\mathrm{Pt}(111)$}

\section{Convergence w.r.t. slab separation}

The activation energies calculated with VASPsol (following the procedure outlined in the previous Section) are found to converge only slowly with respect to the distance $L_{z}$ between periodically repeated slabs. More precisely, the activation energies are a linear function of $1 / L_{z}$, as shown in Figure 2. The slopes of the fitted regression lines appear to be anti-correlated to the difference in $N_{e}$ between the initial and transition states. The slope is namely close to zero for the Tafel reactions (for which $\Delta N_{e} \simeq$ $\left.-0.05 \mathrm{e}^{-}\right)$and negative for the Volmer and Heyrovský reactions (where $\Delta N_{e} \simeq 0.6 \mathrm{e}^{-}$).

The $1 / L_{z}$ dependence suggests the presence of spurious charge interactions in the conventional 3D-periodic implementation in VASPsol. This behavior is possibly connected to the observation that standard Poisson-Boltzmann models do not necessarily enforce strict charge neutrality. ${ }^{54}$ We therefore performed single-point calculations using the JDFTx code which implements a truncated Coulomb scheme to fully decouple periodically repeated slabs. ${ }^{55}$ As shown in Table 1, the JDFTx activation energies indeed agree well with the VASPsol results extrapolated to $L_{z}=\infty$. The small differences that remain can be attributed to the different

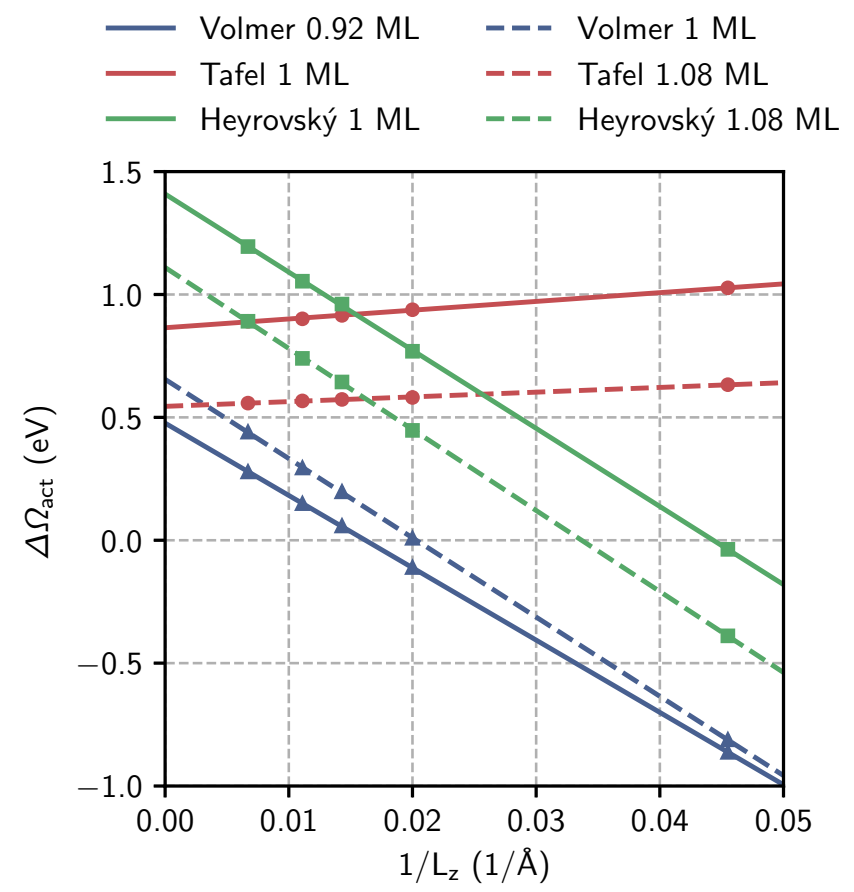

Figure 2: Activation energies calculated using VASPsol for HER reactions on $\mathrm{Pt}(111)$ using Eigen ions as a proton model for the PCET reactions. The values are plotted as function of the inverse of the cell length perpendicular to the plane of the slab. The three types of barriers have been evaluated at two different hydrogen coverages in the reactant state, i.e. at/below $1 \mathrm{ML}$ and above $1 \mathrm{ML}$.

treatments of core-valence interactions and ki- ${ }_{321}$ netic energy cutoffs.

It should furthermore be noted that the ${ }_{323}$ $L_{z}$-dependence seems not to have been taken 324 into account in several previous studies ${ }^{24,25,27}{ }_{325}$ of PCET reactions using the VASPsol code. ${ }^{326}$ Without extrapolation to $L_{z}=\infty$, values of ${ }_{327}$ $L_{z}$ of over $400 \AA$ would be have been required ${ }_{328}$ in the present work to converge the Volmer and 329 Heyrovský barriers on $\mathrm{Pt}(111)$ within $0.1 \mathrm{eV} . \quad{ }_{330}$

\section{Water model}

Figure 3 furthermore shows the barrier heights when an ice bilayer is used for the water structure (red bars), compared to the results with an Eigen ion (blue bars). ${ }^{2}$ The two sets are in fair

\footnotetext{
${ }^{2}$ Note that the Tafel barriers reported in the 'water cluster' approach are calculated without explicit water
} 
Table 1: Comparison of VASPsol activation energies (extrapolated to $L_{z}=\infty$ ) and JDFTx single-point calculations performed at the VASPsol geometries using a Coulomb truncation scheme. Each barrier has been evaluated at two different initial hydrogen coverages $\theta_{\mathrm{H}}$ as well as two different water structures (a water cluster corresponding to the 'Eigen' cation as well as the (protonated) ice bilayer ('IBL') structure).

\begin{tabular}{lccccc}
\hline \hline \multirow{2}{*}{ Reaction } & $\theta_{\mathrm{H}}$ & \multicolumn{4}{c}{$\Delta \Omega_{\mathrm{act}}(\mathrm{eV})$} \\
\hline Volmer & 0.92 & $\begin{array}{c}\text { VASPsol } \\
(\text { Eigen })\end{array}$ & $\begin{array}{c}\text { JDFTx } \\
(\text { Eigen })\end{array}$ & $\begin{array}{c}\text { VASPsol } \\
(\text { IBL })\end{array}$ & $\begin{array}{c}\text { JDFTx } \\
(\text { IBL })\end{array}$ \\
\cline { 3 - 6 } Tafel & 1.00 & 0.477 & 0.479 & 0.692 & 0.693 \\
& 1.00 & 0.865 & 0.629 & 0.805 & 0.838 \\
Heyrovský & 1.08 & 0.545 & 0.407 & 0.420 & 0.416 \\
& 1.00 & 1.408 & 1.364 & 1.618 & 1.625 \\
& 1.08 & 1.110 & 0.995 & 1.393 & 1.388 \\
\hline \hline
\end{tabular}

relevant for $\mathrm{HER}$ on $\mathrm{Pt}(111)$ at $0 \mathrm{~V}_{\mathrm{SHE}}$, and therefore the relative accuracy of the two water models cannot be compared in this context.

Table 2: Hydrogen-bond distances in $\AA$ for the $\mathrm{H}_{3} \mathrm{O}^{+}$ion at the initial state (IS) and transition state (TS) for the Volmer step on $\mathrm{Pt}(111)$ with an initial $\mathrm{H}$ coverage of $0.92 \mathrm{ML}$.

\begin{tabular}{lrr}
\hline \hline & & $\begin{array}{c}\text { Acce- } \\
\text { Water structure }\end{array}$ \\
\hline Water cluster (IS) & $1.39,1.39$ & 2.09 \\
Water cluster (TS) & $1.75,1.80$ & 1.94 \\
Ice bilayer (IS) & $1.45,1.59,1.58$ & - \\
Ice bilayer (TS) & 1.70 & 2.46 \\
\hline \hline
\end{tabular}

Comparison with the extrapolation ap- 369 proach

The calculated barrier heights are now com- 371 pared with literature results obtained with the 372 extrapolation at the same electrode potential, 373 hydrogen coverage, and water model (the ice bi- ${ }_{374}^{374}$ layer structure, see Ref. 6) as also shown in Fig- 375 ure $3 .^{3}$ The agreement is remarkably good, con- 376 sidering the pronounced differences in method- 377

\footnotetext{
${ }^{3}$ In Ref. 6 the Heyrovský barrier at an initial $\mathrm{H}$ coverage of $1 \mathrm{ML}$ is given at $-0.2 \mathrm{~V}_{\mathrm{SHE}}$. We therefore used a typical symmetry factor of 0.5 to estimate the corresponding value at $0 \mathrm{~V}_{\mathrm{SHE}}$.
} 372 3 374 s. . 377 be more accurate than with the cluster model. As will be discussed further below, however, the Volmer and Heyrovský steps are not kinetically molecules. 


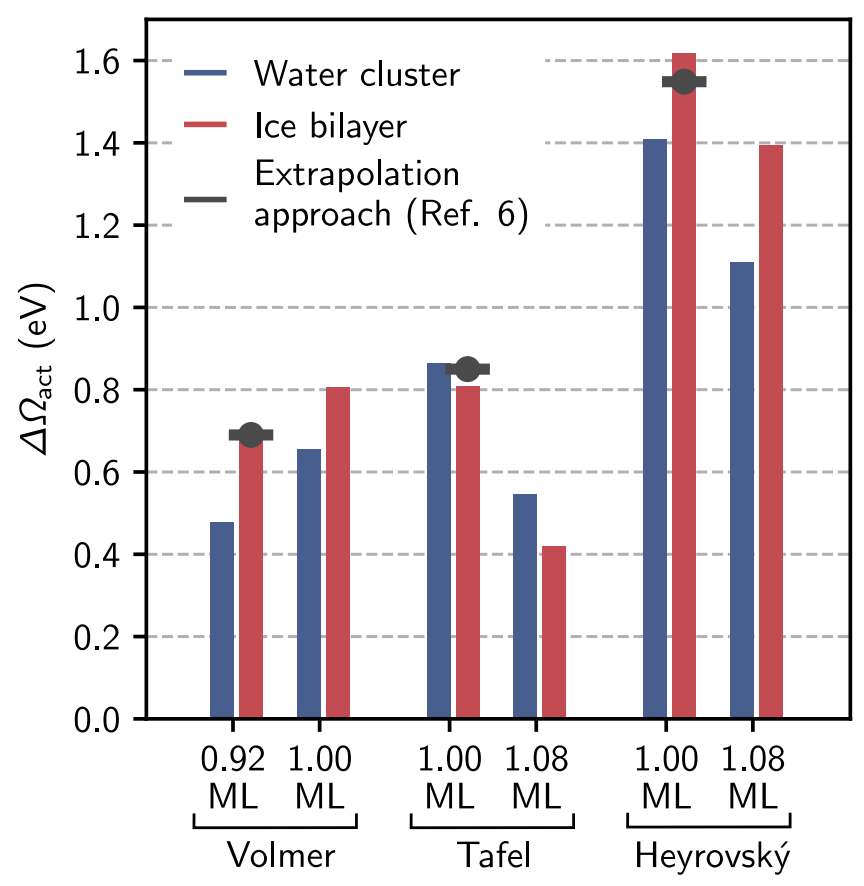

Figure 3: Calculated activation energies for HER reactions on $\mathrm{Pt}(111)$ using the implicit solvation scheme (VASPsol) at different initial hydrogen coverages and with two different water structures. The values obtained via the extrapolation approach are also indicated (see Ref. 6)

ology. Both approaches therefore agree that hydrogen is evolved via a Volmer-Tafel mechanism with the Tafel step as the RDS.

Additionally, Figure 3 shows that with the implicit solvation approach the barriers change as the coverage is increased beyond $1 \mathrm{ML}$, in ways which are similar to the extrapolation approach. ${ }^{6}$ Increasing the coverage beyond $1 \mathrm{ML}$ requires the occupation of the energetically less favorable atop sites. Following a Bell-EvansPolanyi principle, the activation energy for the Volmer step increases, whereas those of the Tafel and Heyrovský steps decrease.

Lastly, the symmetry factors $\beta$ calculated at $0 \mathrm{~V}_{\mathrm{SHE}}$ with the ice bilayer model amount to $0.62\left(\right.$ Volmer, $\left.\theta_{\mathrm{H}}=0.92 \mathrm{ML}\right),-0.04\left(\right.$ Tafel, $\theta_{\mathrm{H}}$ $=1 \mathrm{ML})$, and 0.76 (Heyrovský, $\left.\theta_{\mathrm{H}}=1 \mathrm{ML}\right)$. The activation energies of the two PCET reactions will therefore indeed decrease as the applied potential is lowered, while the barrier for the non-electrochemical Tafel step is nearly potential-independent. Skúlason and cowork- ers $^{6}$ obtained values of $0.44, \sim 0$, and 1.07 , respectively, using the extrapolation approach. These values are, however, not directly comparable to ours, as these also include the potential dependence of the hydrogen coverage and of the transition state geometry and are obtained by linear regression over a fairly wide potential range of $1 \mathrm{~V}$ or more.

\section{Comparison with experimental measure- ments}

Experimental measurements of the HER kinetics on $\mathrm{Pt}(111)$ surfaces are available in the works by Marković et al. ${ }^{35}$ and He et al. ${ }^{60} \mathrm{Al}-$ though both groups measure similar current densities at $0 \mathrm{~V}$, different temperature dependencies are reported. We follow the explanation offered in Ref. 60 that the Pt(111) substrate used in Ref. 35 may have contained low concentrations of highly active defect sites. In this view, the higher apparent activation energy measured by He et al. (circa $0.67 \mathrm{eV}$ ) should be closer to that of a pristine $\mathrm{Pt}(111)$ surface. The magnitude of the corresponding pre-exponential factor (circa $10^{10} \mathrm{~mA} / \mathrm{cm}^{2}$ ) is characteristic of a process involving only surface adsorbates, supporting the Tafel reaction as the RDS. These findings compare well with the computational results described in the previous paragraphs. As the barrier for the Tafel step is not sensitive to the water structure at the interface, this does not, unfortunately, allow to discriminate between different types of water models.

\section{Comparison with other implicit solvent calculations}

Fang et al. have previously addressed ${ }^{31}$ the HER on Pt(111) using a largely similar method implemented in the SIESTA code. ${ }^{32,33}$ At 0 $\mathrm{V}_{\mathrm{SHE}}$ and a hydrogen coverage at or below 1 ML, the Tafel barrier reported by Fang et al. $(0.92 \mathrm{eV})$ agrees well with our calculations, while the reported Volmer and Heyrovský barrier heights are significantly lower than ours $(<0.2 \mathrm{eV}$ and $0.93 \mathrm{eV}$, respectively). We presume the origins of this difference may lie in 
the DFT setup, details of the implicit solvation model, the use of a water trimer cluster (as opposed to the quadrimer employed here), and/or the inclusion of entropic and zero-point vibrational contributions (which are not considered here).

An alternative constant-potential implicit solvation approach has been recently proposed ${ }^{34}$ where essentially the countercharge is not described by a Poisson-Boltzmann equation, but is instead homogeneously spread out over the bulk solvent region. Using this 'solvated jellium method' (SJM), the barrier for the Volmer step on $\operatorname{Pt}(111)$ is found to be significantly lower (circa $0.02 \mathrm{eV}$ at $0 V_{\mathrm{SHE}}$ using the ice bilayer model, compared to $0.7 \mathrm{eV}$ in the present work). Also here further investigation is needed to locate the origin of the discrepancy, i.e. whether or not it is due to the simplified description of the countercharge distribution. One important question is whether the SJM energy differences change as the jellium+solvent width is increased, which entails a progressive dilution of the countercharge concentration.

\section{$\mathrm{H}_{2}$ evolution on $\operatorname{Pt}(110)-(1 \times 2)$}

We now turn to the missing-row reconstructed $\operatorname{Pt}(110)$ surface, with a hydrogen coverage of 1 ML where all ridge and (micro-)facet sites are occupied (see Panel (b) in Figure 4). Although there is a weak thermodynamic preference for the adsorption of additional hydrogen atoms in the trough sites at $U_{\mathrm{SHE}}=0 \mathrm{~V}$ (with differential binding energies of circa $0.1 \mathrm{eV}),{ }^{6,61,62}$ it will be argued below that trough-adsorbed hydrogen atoms, if present, would not contribute significantly to the hydrogen evolution rate at $0 \mathrm{~V}$.

Panel (a) in Figure 4 shows the calculated barrier heights for several relevant Volmer and Tafel steps at and below 1 ML coverage of hydrogen. Various attempts at locating saddle points for the Heyrovský reactions converged to Volmer-like saddle points, suggesting that these reactions are inoperable on this surface. On both sites the Tafel reaction proceeds via an intermediate corresponding to a Kubas complex, ${ }^{63}$ consisting of a stretched hydrogen

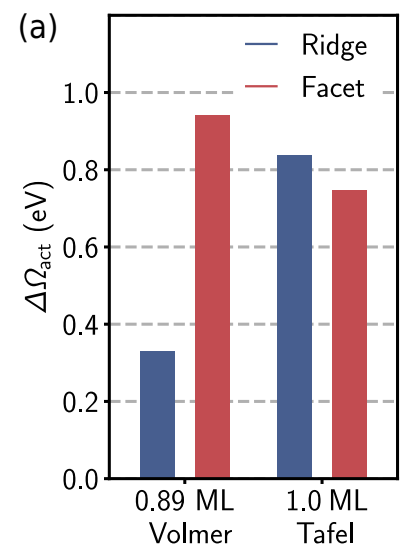

(b)

Figure 4: Panel (a): calculated barrier heights for the Volmer and Tafel processes on the Pt(110)- $(1 \times 2)$ surface. Panel (b): structural model of the transition state for the Volmer reaction on a (micro-)facet.

molecule on top of a platinum atom (see Figure 5). Desorption of $\mathrm{H}_{2}$ via the Tafel reaction is faster on the facet $(0.75 \mathrm{eV})$ compared to the ridge $(0.84 \mathrm{eV})$. This is in line with the higher binding energy of the ridge-bound adatoms. These results are comparable to calculations in the literature using the same functional and hydrogen coverage, but without solvent and at the potential of zero charge. ${ }^{6,62}$
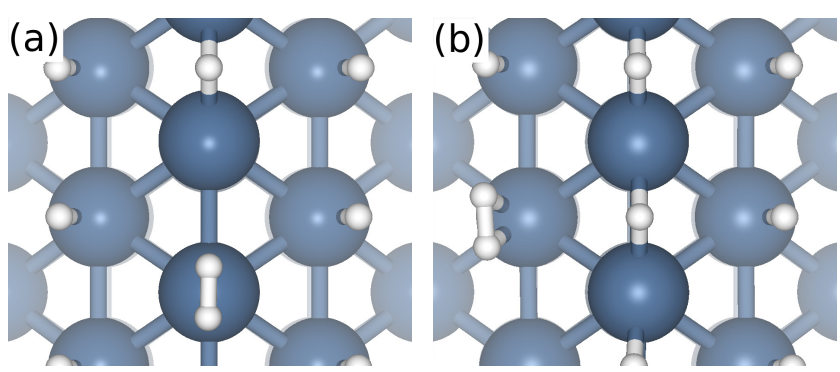

Figure 5: Structural models of the Kubas complex as an intermediate of the Tafel reaction on ridges (a) and microfacets (b) of the $\mathrm{Pt}(110)$ $(1 \times 2)$ surface.

Similarly, at an initial hydrogen coverage of ${ }_{501}$ 0.89 ML, we find a higher barrier for the Volmer $\quad 502$ step on the facets $(0.94 \mathrm{eV})$ compared to the ${ }_{503}$ ridge $(0.33 \mathrm{eV})$. This change exceeds the differ- ${ }_{504}$ ence in hydrogen binding energy between the ${ }_{505}$ two site types (calculated to be only $0.23 \mathrm{eV}$ ). ${ }_{506}$ Hence, even though hydrogen desorption will 507 take place on the facets, replenishment of the 508 coverage happens most rapidly via diffusion of 509 


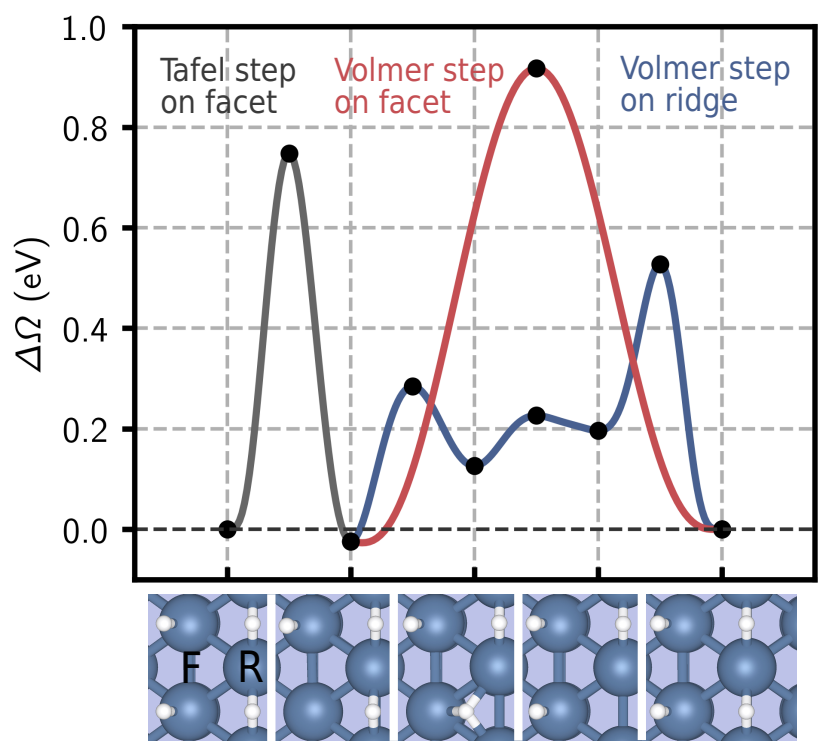

Figure 6: Calculated energy diagram for HER on $\mathrm{Pt}(110)-(1 \times 2)$ for a steady-state hydrogen coverage of $1 \mathrm{ML}$. The reaction energies have been evaluated using the CHE formalism.

$\mathrm{H}$ atoms from the ridge to the vacant facet sites followed by Volmer discharge on the nowvacant ridge site. This process is illustrated in the energy diagram in Figure 6. As the diffusion barrier is low $(0.3 \mathrm{eV})$, the Volmer step will dictate the apparent activation energy, which then amounts to $0.56 \mathrm{eV}$. This entails that hydrogen deposition is fast relative to the Tafel reaction, making the latter the ratedetermining step. This mechanism, as well as the lower barrier of the rate-limiting Tafel step compared to Pt(111), is in good agreement with the measurements on Pt single crystal surfaces by Marković and coworkers. ${ }^{35}$

We now return to the question whether hydrogen coverages beyond $1 \mathrm{ML}$ (with occupations of trough sites) could be relevant at $U=0 \mathrm{~V}_{\mathrm{SHE}}$. Firstly, the relatively high barrier obtained for the Volmer reaction on the facet suggests that the corresponding barrier on the trough will be even higher, requiring a similar diffusion-assisted mechanism as described above. However, moving a ridge-bound $\mathrm{H}$ atom to a trough position requires more energy $(0.46$ $\mathrm{eV}$ ) than to a facet site. Achieving a significant occupation of the trough sites is, therefore, kinetically difficult. Additionally, even if a partial occupation of the trough sites would be reached, the Tafel reaction on the facets would ${ }_{538}$ remain the main desorption pathway for hydro- ${ }_{539}$ gen. Previous studies have namely found that, 540 despite the lower binding energy, the barrier for ${ }_{541}$ hydrogen desorption from trough sites is larger 542 compared to the facet sites when the hydro- ${ }_{543}$ gen coverage is at or slightly beyond 1 ML. ${ }^{61,62}{ }_{544}^{544}$ It is therefore sufficient to only consider ridge- 545 and facet-bound hydrogen atoms for HER on 546 $\operatorname{Pt}(110)-(1 \times 2)$ at $0 \mathrm{~V}_{\mathrm{SHE}}$.

\section{Conclusions}

We have evaluated the performance of a recently developed constant-potential implicit solvation approach to electrochemical barriers for one of the classical processes in catalysis, the hydrogen evolution reaction on platinum. The relevant Volmer, Tafel, and Heyrovský kinetics on $\mathrm{Pt}(111)$ and $\mathrm{Pt}(110)$ at $0 \mathrm{~V}$ versus SHE compare well with the previously developed 'extrapolation' approach and with available experimental data. Importantly, the inclusion of an implicit solvent only moderately increases the computational cost, whereas calculating barrier heights with the extrapolation approach is more expensive by at least one order of magnitude. Care should be taken, however, in dealing with spurious electrostatic interactions between periodically repeated slabs when using the VASPsol code.

Although further testing for other types of reactions and materials is warranted, we have so far found the accuracy to be satisfactory and expect implicit solvation approaches to receive increasing attention in future research. The application and continued development of costeffective methods is most welcome, as the previous lack of such approaches has frequently led to kinetic aspects being disregarded in firstprinciples electrocatalysis research.

Acknowledgement The authors thank Vitae Industries for providing computational resources, and Javed Hussain for valuable discussions. This material is based upon work supported by the National Science Foundation under Grant No. CHE-1665372.

549

550 551 552 553 554 555 556 557 558 559 560 561 562 563 564 565 566 567 568 569 570 571 572 573 574 575 576 
Supporting Information Available

Coordinate files pertaining to the hydrogen evolution reactions on $\mathrm{Pt}(111)$ and $\mathrm{Pt}(110)$.

\section{References}

(1) Nørskov, J. K.; Rossmeisl, J.; Logadottir, A.; Lindqvist, L.; Kitchin, J. R.; Bligaard, T.; Jónsson, H. Origin of the Overpotential for Oxygen Reduction at a FuelCell Cathode. J. Phys. Chem. B 2004, 108, 17886-17892.

(2) Nørskov, J. K.; Bligaard, T.; Logadottir, A.; Kitchin, J. R.; Chen, J. G.; Pandelov, S.; Stimming, U. Trends in the Exchange Current for Hydrogen Evolution. J. Electrochem. Soc. 2005, 152, J23-J26.

(3) Skúlason, E.; Jónsson, H. Atomic scale simulations of heterogeneous electrocatalysis: recent advances. Adv. Phys. X 2017, 2, 481-495.

(4) Exner, K. S.; Over, H. Kinetics of Electrocatalytic Reactions from First-Principles: A Critical Comparison with the Ab Initio Thermodynamics Approach. Acc. Chem. Res. 2017, 50, 1240-1247.

(5) Rossmeisl, J.; Skúlason, E.; Björketun, M. E.; Tripkovic, V.; Nørskov, J. K. Modeling the electrified solid-liquid interface. J. Chem. Phys. Lett. 2008, 466, 68-71.

(6) Skúlason, E.; Tripkovic, V.; Björketun, M. E.; Gudmundsdóttir, S.; Karlberg, G.; Rossmeisl, J.; Bligaard, T.; Jónsson, H.; Nørskov, J. K. Modeling the Electrochemical Hydrogen Oxidation and Evolution Reactions on the Basis of Density Functional Theory Calculations. J. Phys. Chem. C 2010, 114, 18182-18197.

(7) Skúlason, E.; S. Karlberg, G.; Rossmeisl, J.; Bligaard, T.; Greeley, J.;
Jónsson, H.; K. Nørskov, J. Density func- ${ }_{617}$ tional theory calculations for the hydro- 618 gen evolution reaction in an electrochemi- ${ }_{619}$ cal double layer on the $\operatorname{Pt}(111)$ electrode. ${ }_{620}$ Phys. Chem. Chem. Phys. 2007, 9, 3241- ${ }_{621}$ 3250 .

(8) Hussain, J.; Jónsson, H.; Skúlason, E. ${ }_{623}$ Faraday efficiency and mechanism of elec- ${ }_{624}$ trochemical surface reactions: $\mathrm{CO}_{2}$ reduc- ${ }_{625}$ tion and $\mathrm{H}_{2}$ formation on $\mathrm{Pt}(111)$. Fara- ${ }_{626}$ day Discuss. 2016, 195, 619-636.

(9) Hussain, J.; Jónsson, H.; Skúlason, E. Cal- ${ }_{628}$ culations of Product Selectivity in Elec- ${ }_{629}$ trochemical $\mathrm{CO}_{2}$ Reduction. ACS Catal. ${ }_{630}$ 2018, 5240-5249.

(10) Chan, K.; Nørskov, J. K. Electrochemi- ${ }_{632}$ cal Barriers Made Simple. J. Phys. Chem. ${ }_{633}$ Lett. 2015, 6, 2663-2668.

(11) Chan, K.; Nørskov, J. K. Potential Depen- ${ }_{635}$ dence of Electrochemical Barriers from ab ${ }_{636}$ Initio Calculations. J. Phys. Chem. Lett. ${ }_{637}$ 2016, 7, 1686-1690.

(12) Filhol, J.-S.; Neurock, M. Elucidation of ${ }_{639}$ the Electrochemical Activation of Water ${ }_{640}$ over Pd by First Principles. Angew. Chem. ${ }_{641}$ Int. Ed. 2006, 45, 402-406.

(13) Taylor, C. D.; Wasileski, S. A.; Filhol, J.- ${ }_{643}$ S.; Neurock, M. First principles reaction 644 modeling of the electrochemical interface: ${ }_{645}$ Consideration and calculation of a tunable ${ }_{646}$ surface potential from atomic and elec- ${ }_{647}$ tronic structure. Phys. Rev. B 2006, 73, 648 165402 .

(14) Makov, G.; Payne, M. C. Periodic bound- ${ }_{650}$ ary conditions in ab initio calculations. ${ }_{651}$ Phys. Rev. B 1995, 51, 4014-4022. 652

(15) Komsa, H.-P.; Rantala, T. T.; 653 Pasquarello, A. Finite-size supercell 654 correction schemes for charged defect 655 calculations. Phys. Rev. B 2012, 86, 656 045112 .

(16) Otani, M.; Hamada, I.; Sugino, O.; 658 Morikawa, Y.; Okamoto, Y.; Ikeshoji, T. 659 
Structure of the water/platinum interface - a first principles simulation under bias potential. Phys. Chem. Chem. Phys. 2008, 10, 3609-3612.

(17) Surendralal, S.; Todorova, M.; Finnis, M. W.; Neugebauer, J. FirstPrinciples Approach to Model Electrochemical Reactions: Understanding the Fundamental Mechanisms behind $\mathrm{Mg}$ Corrosion. Phys. Rev. Lett. 2018, 120, 246801.

(18) Akhade, S. A.; Bernstein, N. J.; Esopi, M. R.; Regula, M. J.; Janik, M. J. A simple method to approximate electrode potential-dependent activation energies using density functional theory. Catal. Today 2017, 288, 63-73.

(19) Akhade, S. A.; Nidzyn, R. M.; Rostamikia, G.; Janik, M. J. Using BrønstedEvans-Polanyi relations to predict electrode potential-dependent activation energies. Catal. Today 2018, 312, 82-91.

(20) Sundararaman, R.; Goddard, W. A.; Arias, T. A. Grand canonical electronic density-functional theory: Algorithms and applications to electrochemistry. J. Chem. Phys. 2017, 146, 114104.

(21) Sundararaman, R.; LetchworthWeaver, K.; Schwarz, K. A.; Gunceler, D.; Ozhabes, Y.; Arias, T. A. JDFTx: Software for joint density-functional theory. SoftwareX 2017, 6, 278-284.

(22) Mathew, K.; Sundararaman, R.; Letchworth-Weaver, K.; Arias, T. A.; Hennig, R. G. Implicit solvation model for density-functional study of nanocrystal surfaces and reaction pathways. $J$. Chem. Phys. 2014, 140, 084106.

(23) Mathew, K.; Hennig, R. G. Implicit self-consistent description of electrolyte in plane-wave density-functional theory. arXiv:1601.03346 [cond-mat] 2016, arXiv: 1601.03346.
(24) Goodpaster, J. D.; Bell, A. T.; Head- 703 Gordon, M. Identification of Possible 704 Pathways for C-C Bond Formation during 705 Electrochemical Reduction of $\mathrm{CO}_{2}$ : New 706 Theoretical Insights from an Improved 707 Electrochemical Model. J. Phys. Chem. 708 Lett. 2016, 7, 1471-1477.

(25) Singh, M. R.; Goodpaster, J. D.; We- 710 ber, A. Z.; Head-Gordon, M.; Bell, A. T. ${ }_{711}$ Mechanistic insights into electrochemical 712 reduction of $\mathrm{CO}_{2}$ over $\mathrm{Ag}$ using density ${ }_{713}$ functional theory and transport models. 714 PNAS 2017, 114, E8812-E8821. 715

(26) Garza, A. J.; Bell, A. T.; Head- 716 Gordon, M. Is Subsurface Oxygen Neces- 717 sary for the Electrochemical Reduction of 718 $\mathrm{CO}_{2}$ on Copper? J. Phys. Chem. Lett. ${ }^{719}$ 2018, 9, 601-606.

(27) Garza, A. J.; Bell, A. T.; Head- ${ }_{721}$ Gordon, M. Mechanism of $\mathrm{CO}_{2}$ Reduction 722 at Copper Surfaces: Pathways to $\mathrm{C}_{2}$ Prod- ${ }_{723}$ ucts. ACS Catal. 2018, 8, 1490-1499. 724

(28) Schwarz, K. A.; Sundararaman, R.; Mof- 725 fat, T. P.; Allison, T. C. Formic acid ox- 726 idation on platinum: a simple mechanis- ${ }_{727}$ tic study. Phys. Chem. Chem. Phys. 2015, ${ }_{728}$ 17, 20805-20813.

(29) Wang, H.-F.; Liu, Z.-P. Formic Acid Oxi- 730 dation at $\mathrm{Pt} / \mathrm{H} 2 \mathrm{O}$ Interface from Periodic 731 DFT Calculations Integrated with a Con- 732 tinuum Solvation Model. J. Phys. Chem. 733 C 2009, 113, 17502-17508.

(30) Fang, Y.; Liu, Z. Electrochemical reac- 735 tions at the electrode/solution interface: 736 Theory and applications to water elec- ${ }_{737}$ trolysis and oxygen reduction. Sci. China 738 Chem. 2010, 53, 543-552.

(31) Fang, Y.-H.; Wei, G.-F.; Liu, Z.-P. Cat- 740 alytic Role of Minority Species and Mi- 741 nority Sites for Electrochemical Hydro- 742 gen Evolution on Metals: Surface Charg- 743 ing, Coverage, and Tafel Kinetics. J. Phys. 744 Chem. C 2013, 117, 7669-7680. 
(32) Fang, Y.-H.; Wei, G.-F.; Liu, Z.-P. Theoretical modeling of electrode/electrolyte interface from first-principles periodic continuum solvation method. Catal. Today 2013, 202, 98-104.

(33) Fang, Y.-H.; Liu, Z.-P. Tafel Kinetics of Electrocatalytic Reactions: From Experiment to First-Principles. ACS Catal. 2014, 4, 4364-4376.

(34) Kastlunger, G.; Lindgren, P.; Peterson, A. A. Controlled-Potential Simulation of Elementary Electrochemical Reactions: Proton Discharge on Metal Surfaces. J. Phys. Chem. C 2018,

(35) Marković, N. M.; Grgur, B. N.; Ross, P. N. Temperature-Dependent Hydrogen Electrochemistry on Platinum Low-Index Single-Crystal Surfaces in Acid Solutions. J. Phys. Chem. B 1997, 101, 5405-5413.

(36) Kresse, G.; Hafner, J. Ab Initio MolecularDynamics Simulation of the Liquid-MetalAmorphous-Semiconductor Transition in Germanium. Phys. Rev. B 1994, 49, 14251-14269.

(37) Kresse, G.; Furthmüller, J. Efficiency of Ab-Initio Total Energy Calculations for Metals and Semiconductors Using a Plane-Wave Basis Set. Comp. Mater. Sci. 1996, $6,15-50$.

(38) Kresse, G.; Furthmüller, J. Efficient Iterative Schemes for Ab Initio Total-Energy Calculations Using a Plane-Wave Basis Set. Phys. Rev. B 1996, 54, 11169-11186.

(39) Kresse, G.; Joubert, D. From ultrasoft pseudopotentials to the projector augmented-wave method. Phys. Rev. B 1999, 59, 1758-1775.

(40) Hohenberg, P.; Kohn, W. Inhomogeneous Electron Gas. Phys. Rev. 1964, 136, B864-B871.

(41) Kohn, W.; Sham, L. J. Self-Consistent Equations Including Exchange and Correlation Effects. Phys. Rev. 1965, 140, A1133-A1138.
(42) Garrity, K. F.; Bennett, J. W.; 790 Rabe, K. M.; Vanderbilt, D. Pseu- 791 dopotentials for high-throughput DFT 792 calculations. Comp. Mater. Sci. 2014, 81, 793 446-452.

(43) Hammer, B.; Hansen, L. B.; 795 Nørskov, J. K. Improved adsorption 796 energetics within density-functional 797 theory using revised Perdew-Burke- 798 Ernzerhof functionals. Phys. Rev. $B \quad 799$ 1999, 59, 7413-7421.

(44) Perdew, J. P.; Burke, K.; Ernzer- 801 hof, M. Generalized Gradient Approxima- 802 tion Made Simple. Phys. Rev. Lett. 1996, 803 7r, 3865-3868.

(45) Marques, M. A. L.; Oliveira, M. J. T.; 805 Burnus, T. Libxc: A library of ex- 806 change and correlation functionals for den- 807 sity functional theory. Computer Physics 808 Communications 2012, 183, 2272-2281. 809

(46) Monkhorst, H. J.; Pack, J. D. Spe- 810 cial points for Brillouin-zone integrations. 811 Phys. Rev. B 1976, 13, 5188-5192. 812

(47) Pack, J. D.; Monkhorst, H. J. 'Special ${ }_{813}$ points for Brillouin-zone integrations' - a 814 reply. Phys. Rev. B 1977, 16, 1748-1749. 815

(48) Henkelman, G.; Jónnson, H. Improved 816 tangent estimate in the nudged elastic 817 band method for finding minimum energy 818 paths and saddle points. J. Chem. Phys. ${ }_{819}$ 2000, 113, 9978-9985.

(49) Henkelman, G.; Jónsson, H. A dimer 821 method for finding saddle points on high 822 dimensional potential surfaces using only 823 first derivatives. J. Chem. Phys. 1999, 824 111, 7010-7022.

(50) Gunceler, D.; Letchworth-Weaver, K.; 826 Sundararaman, R.; Schwarz, K. A.; 827 Arias, T. A. The importance of nonlinear 828 fluid response in joint density-functional 829 theory studies of battery systems. Mod- 830 elling Simul. Mater. Sci. Eng. 2013, 21, 831 074005 . 
(51) Andreussi, O.; Dabo, I.; Marzari, N. Revised self-consistent continuum solvation in electronic-structure calculations. $J$. Chem. Phys. 2012, 136, 064102.

(52) Murrell, J.; Jenkins, A. Properties of liquids and solutions, 2nd ed.; Wiley \& Sons: Chichester, England, 1994.

(53) Trasatti, S. The absolute electrode potential: an explanatory note (Recommendations 1986). Pure Appl. Chem. 1986, 58, 955-966.

(54) Melander, M.; Kuisma, M.; Christensen, T.; Honkala, K. Grand-Canonical Approach to Density Functional Theory of Electrocatalytic Systems: Thermodynamics of Solid-Liquid Interfaces at Constant Ion and Electrode Potentials; ChemRxiv, 2018.

(55) Sundararaman, R.; Arias, T. A. Regularization of the Coulomb singularity in exact exchange by Wigner-Seitz truncated interactions: Towards chemical accuracy in nontrivial systems. Phys. Rev. B 2013, $87,165122$.

(56) Meng, S.; Xu, L. F.; Wang, E. G.; Gao, S. Vibrational Recognition of HydrogenBonded Water Networks on a Metal Surface. Phys. Rev. Lett. 2002, 89, 176104.

(57) Ogasawara, H.; Brena, B.; Nordlund, D.; Nyberg, M.; Pelmenschikov, A.; Pettersson, L. G. M.; Nilsson, A. Structure and Bonding of Water on Pt(111). Phys. Rev. Lett. 2002, 89, 276102.

(58) Schnur, S.; Groß, A. Properties of metalwater interfaces studied from first principles. New J. Phys. 2009, 11, 125003.

(59) Groß, A.; Gossenberger, F.; Lin, X.; Naderian, M.; Sakong, S.; Roman, T. Water Structures at Metal Electrodes Studied by Ab Initio Molecular Dynamics Simulations. J. Electrochem. Soc. 2014, 161, E3015-E3020.
(60) He, Z.-D.; Wei, J.; Chen, Y.-X.; San- 875 tos, E.; Schmickler, W. Hydrogen evolu- 876 tion at $\mathrm{Pt}(111)$ - activation energy, fre- 877 quency factor and hydrogen repulsion. 878 Electrochim. Acta 2017, 255, 391-395.

(61) Gudmundsdóttir, S.; Skúlason, E.; 880 Jónsson, H. Reentrant Mechanism for As- 881 sociative Desorption: $\mathrm{H}_{2} / \mathrm{Pt}(110)-(1 \times 2) . \quad 882$ Phys. Rev. Lett. 2012, 108, 156101.

(62) Gudmundsdóttir, S.; Skúlason, E.; West- 884 strate, K.-J.; Juurlink, L.; Jónsson, H. 885 Hydrogen adsorption and desorption at 886 the $\mathrm{Pt}(110)-(1 \times 2)$ surface: experimental ${ }_{887}$ and theoretical study. Phys. Chem. Chem. ${ }_{888}$ Phys. 2013, 15, 6323-6332.

(63) Kubas, G. J. Molecular hydrogen com- 890 plexes: coordination of a $\sigma$ bond to tran- 891 sition metals. Acc. Chem. Res. 1988, 21, 892 120-128. 
${ }_{894}$ Graphical TOC Entry
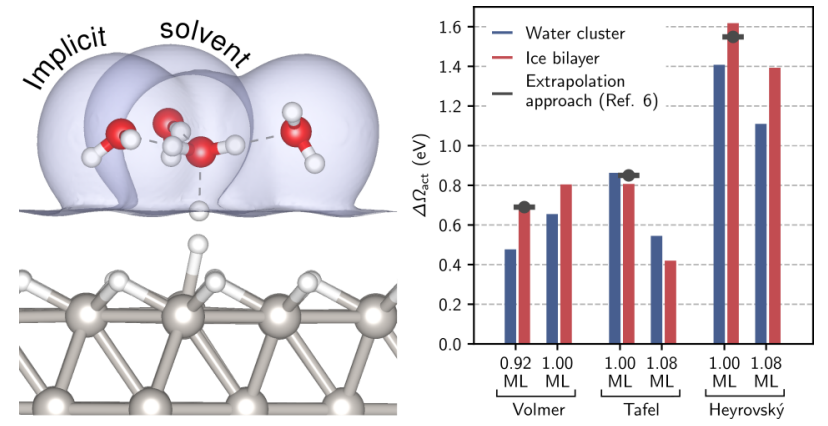